\title{
Collective dynamics in highly relativistic heavy-ion collisions*
}

\author{
A. Dumitru \\ Physics Department, Yale University \\ P.O. Box 208124, New Haven, CT 06520, USA \\ D.H. Rischke \\ RIKEN-BNL Research Center, Brookhaven National Laboratory \\ Upton, NY 11973, USA
}

(January 16, 2018)

\begin{abstract}
Hydrodynamics with cylindrical symmetry in transverse direction and longitudinal scaling flow is employed to calculate the transverse momentum spectra of various hadrons and clusters (e.g. $\pi, K, N, \Phi, \Lambda, d, H e$ ) in central heavy-ion collisions at CERN-SPS and BNL-RHIC energies up to $p_{T}=4 \mathrm{GeV}$. We discuss the sensitivity of these spectra with respect to the initial transverse density profile as well as to the choice of "freeze-out" hypersurface. For $\sqrt{s}=18 A \mathrm{GeV}$ (SPS energy) and $p_{T}<2 \mathrm{GeV}$, overall good agreement of the $p_{T}$ distributions with data is found when freeze-out occurs along the $T=130 \mathrm{MeV}$ isotherm. Even high- $p_{T}$ neutral pion data can be described for a particular choice of the initial transverse density profile. It is shown that the average transverse velocity $\left\langle v_{T}\right\rangle$ of heavy hadrons and hadronic clusters is a good measure for the collective flow velocity. The latter is found to be rather similar for SPS and RHIC energies, due to the "stall" of the flow within the long-lived mixed phase at RHIC. In case of thermalization and hydrodynam-
\end{abstract}

\footnotetext{
*Supported by the German Academic Exchange Service (DAAD).
} 
ical expansion, the mean transverse momentum $\left\langle p_{T}\right\rangle$ increases linearly with the hadron mass. In contrast, the string model FRITIOF 7.02, which does not account for rescattering of secondary hadrons, predicts a strong dependence of $\left\langle p_{T}\right\rangle$ on the quark composition of the hadron. Due to the different sensitivity to hard processes, hadrons with charm (anti-)quarks acquire significantly more transverse momentum than hadrons without $c$ (or even without s) quarks.

\section{INTRODUCTION}

A variety of experimental data for rapidity and transverse momentum spectra of various hadrons and clusters [15] in relativistic heavy-ion collisions at the BNL-AGS and CERNSPS indicate significant collective (longitudinal and transverse) expansion of the produced hot and dense fireball [6 13]. Also at the much higher center-of-mass energy that will be reached at the BNL-RHIC, it is commonly expected that the parton density at midrapidity in central collisions is high enough to lead to rapid thermalization 14 16.

In the present paper we discuss the collective evolution of the system following thermalization within a hydrodynamical model. We compute the transverse momentum spectra at midrapidity $d N /\left.d^{2} p_{T} d y\right|_{y=0}$, the mean transverse momenta $\left\langle p_{T}\right\rangle$, and the mean transverse velocities $\left\langle v_{T}\right\rangle$ of various hadron species for $P b+P b$ at CERN-SPS energy $(\sqrt{s}=18 A \mathrm{GeV}$, entropy per net baryon $\left.s / \rho_{B} \approx 40\right)$ and $A u+A u$ at BNL-RHIC energy $(\sqrt{s}=200 A \mathrm{GeV}$, $\left.s / \rho_{B} \approx 200\right)$. Finally, we also calculate the $\left\langle p_{T}\right\rangle$ of various hadrons in $A u+A u$ at RHIC

energy within a string model without rescattering of produced particles (FRITIOF 7.02 [17]) and compare its predictions to those of hydrodynamics. 


\section{MODEL DESCRIPTION}

\section{A. Scaling Hydrodynamics}

Hydrodynamics is defined by (local) energy-momentum and net charge conservation [18],

$$
\partial_{\mu} T^{\mu \nu}=0 \quad, \quad \partial_{\mu} N_{i}^{\mu}=0
$$

$T^{\mu \nu}$ denotes the energy-momentum tensor, and $N_{i}^{\mu}$ the net four-current of the $i$ th conserved charge. We will explicitly consider only one such conserved charge, the net baryon number. We implicitly assume that all other charges which are conserved on strong-interaction time scales, e.g. strangeness, charm, and electric charge, vanish locally. The corresponding fourcurrents are therefore identically zero, cf. eq. (2), and the conservation equations are trivial.

For ideal fluids, the energy-momentum tensor and the net baryon current assume the simple form 18

$$
T^{\mu \nu}=(\epsilon+p) u^{\mu} u^{\nu}-p g^{\mu \nu} \quad, \quad N_{B}^{\mu}=\rho_{B} u^{\mu}
$$

where $\epsilon, p, \rho_{B}$ are energy density, pressure, and net baryon density in the local rest frame

of the fluid, which is defined by $N_{B}^{\mu}=\left(\rho_{B}, \overrightarrow{0}\right) \cdot g^{\mu \nu}=\operatorname{diag}(+,-,-,-)$ is the metric tensor, and $u^{\mu}=\gamma(1, \vec{v})$ the four-velocity of the fluid $\left(\vec{v}\right.$ is the three-velocity and $\gamma=\left(1-\vec{v}^{2}\right)^{-1 / 2}$ the Lorentz factor). The system of partial differential equations (1) is closed by choosing an equation of state $(\mathrm{EoS})$ in the form $p=p\left(\epsilon, \rho_{B}\right)$, cf. below.

For simplicity, we assume cylindrically symmetric transverse expansion with a longitudinal scaling flow profile, $v_{z}=z / t[19]$. At $z=0$, equations (11) reduce to

$$
\partial_{t} E+\partial_{T}\left[(E+p) v_{T}\right]=-\left(\frac{v_{T}}{r_{T}}+\frac{1}{t}\right)(E+p)
$$




$$
\begin{aligned}
\partial_{t} M+\partial_{T}\left(M v_{T}+p\right) & =-\left(\frac{v_{T}}{r_{T}}+\frac{1}{t}\right) M, \\
\partial_{t} R+\partial_{T}\left(R v_{T}\right) & =-\left(\frac{v_{T}}{r_{T}}+\frac{1}{t}\right) R,
\end{aligned}
$$

where we defined $E \equiv T^{00}, M \equiv T^{0 T}$, and $R \equiv N_{B}^{0}$. In the above expressions, the index $T$ refers to the transverse component of the corresponding quantity.

The set of equations (3) describes the evolution in the $z=0$ plane. Due to the assumption of longitudinal scaling, the solution at any other $z \neq 0$ can be simply obtained by a Lorentz boost. The above equations also imply

$$
\left.\frac{\partial p}{\partial \eta}\right|_{\tau, r_{T}}=0
$$

where $\eta \equiv \operatorname{Artanh} v_{z}$ and $\tau \equiv \sqrt{t^{2}-z^{2}}$. This means that on proper-time hyperbolas pressure gradients in rapidity direction vanish, and there is no flow between adjacent infinitesimal rapidity slices. However, only for net baryon free matter, $\rho_{B} \equiv 0$, does this automatically also mean that the temperature $T$ is independent of the longitudinal fluid rapidity $\eta$. In the case $\rho_{B} \neq 0$ equation (雨) only demands

$$
\left.s \frac{\partial T}{\partial \eta}\right|_{\tau}+\left.\rho_{B} \frac{\partial \mu_{B}}{\partial \eta}\right|_{\tau}=0
$$

$s$ and $\mu_{B}$ denote entropy density and baryo-chemical potential, respectively. If other charges like strangeness or electric charge are locally nonvanishing, additional terms appear. Equation (5) does not imply that the rapidity distribution of produced particles is flat (i.e. independent of rapidity) or that the rapidity distributions of various species of hadrons, e.g. pions, kaons, and nucleons, are similar. Any rapidity-dependent $T$ and $\mu_{B}$ that satisfy eq. (5) are in agreement with energy-momentum and net baryon number conservation, as well as with longitudinal scaling flow $v_{z}=z / t$. In this paper, however, we do not explore the ra- 
pidity dependence of the particle spectra, and thus, as discussed below, are not required to specify $T$ and $\mu_{B}$ as functions of $\eta$.

The hydrodynamical equations of motion are solved on a discretized space-time grid $\left(\Delta r_{T}=R_{T} / 100=0.06 \mathrm{fm}, \Delta \tau=0.99 \Delta r_{T}\right)$ employing the RHLLE algorithm as described and tested in 20,21. We have checked that the algorithm conserves total energy and baryon number to within $1 \%$ and that profiles of rarefaction and shock waves are reproduced accurately for various initial conditions [21,22].

\section{B. Equation of State}

To close the system of coupled equations of hydrodynamics, an equation of state (EoS) has to be specified. Lattice QCD predicts a phase transition from ordinary nuclear matter to a so-called quark-gluon plasma (QGP) at a critical temperature of $T_{C}=140-160 \mathrm{MeV}$ [23] (for $\rho_{B}=0$ ). We model the high-temperature phase as an ideal gas of $u, d, s$ quarks (with masses $m_{u}=m_{d}=0, m_{s}=150 \mathrm{MeV}$ ), and gluons, employing the well-known MIT bag model EoS [24]. In the low-temperature region we assume an ideal hadron gas that includes the well-established (strange and nonstrange) hadrons up to masses of $2 \mathrm{GeV}$ (as listed in 25]). The phase coexistence region is constructed employing Gibbs' conditions of phase equilibrium. The bag parameter of $B=380 \mathrm{MeV} / \mathrm{fm}^{3}$ is chosen to yield the critical temperature $T_{C} \approx 160 \mathrm{MeV}$.

\section{Initial Conditions}

Equation (4) suggests that it is convenient to specify the initial conditions in scaling hydrodynamics on a proper-time hyperbola $\tau=\tau_{i}$. Apart from $\tau_{i}$, we also have to specify the 
entropy per net baryon, $s / \rho_{B}$, and the net baryon rapidity density at midrapidity, $d N_{B} / d y$. The initial energy and net baryon densities as well as the temperature and the chemical potentials are then determined by the EoS. After specifying an initial transverse density distribution, the solution of relativistic ideal hydrodynamics is uniquely determined in the forward lightcone.

For collisions at SPS energy we assume that hydrodynamic flow starts at proper time $\tau_{i}=1 \mathrm{fm} / \mathrm{c}$. This is a value conventionally assumed in the literature, cf. e.g. [19]. Note that the production time of the partons in the central region might be smaller, $\simeq 0.2-0.3 \mathrm{fm}$, cf. e.g. [26]. However, we assume that one to two further collisions per particle are necessary for thermalization and onset of collective behavior.

We further assume $d N_{B} / d y=80$, which (after resonance decays) results in $\approx 60$ net nucleons at midrapidity. If we simply scale by $1 / 2$ (at high energy the initial isospin asymmetry is transferred to the pions and the nucleons become almost isospin symmetric), the net proton multiplicity at midrapidity agrees with preliminary NA49 data [1].

The specific entropy in central heavy-ion collisions at SPS energies is $s / \rho_{B}=40-50$, cf. e.g. refs. [4, 27,28] and references therein. With this entropy per net baryon most hadron multiplicities as measured at CERN-SPS can be well described, e.g. the multiplicity of pions in central $\mathrm{Pb}+\mathrm{Pb}$ at midrapidity, $d N_{\pi} / d y \approx 600$ [1].

The initial energy and net baryon densities follow as $\epsilon_{i}=5.3 \mathrm{GeV} / \mathrm{fm}^{3}$ and $\rho_{B}=4.5 \rho_{0}$, respectively. The initial temperature and quark-chemical potentials are $T_{i}=216 \mathrm{MeV}$, and $\mu_{q}=167 \mathrm{MeV}, \mu_{s}=0$, respectively. An estimate of the initial energy density could also be obtained using the Bjorken formula

$$
\epsilon_{i}=\frac{d E_{T} / d y}{\tau_{i} \pi R_{T}^{2}} \approx 3 \mathrm{GeV} / \mathrm{fm}^{3}
$$


where $d E_{T} / d y=405 \mathrm{GeV}$ [29] is the measured transverse energy in the most central $\mathrm{Pb}+\mathrm{Pb}$ events. Since this simple formula does not account for the work performed by expansion, cf. e.g. [10], it predicts an initial energy density that is almost a factor of two below that assumed in our calculation. We obtain $d E_{T} / d y=412 \mathrm{GeV}$ on the hadronization hypersurface, and $d E_{T} / d y=406 \mathrm{GeV}$ on the $T=130 \mathrm{MeV}$ isotherm.

Let us now turn to the initial conditions for central $A u+A u$ collisions at RHIC energy. Due to the higher parton density at midrapidity (as compared to collisions at SPS energy), thermalization may be reached earlier at RHIC. According to various studies [14] [16], thermalization might occur within $\sim 0.5 \mathrm{fm}$. Following [20] we will assume $\tau_{i}=R_{T} / 10=0.6 \mathrm{fm}$ for $A u$ nuclei.

Various microscopic models, e.g. PCM [15], RQMD 1.07 [30], FRITIOF 7.02 [31], and HIJING/B [32], predict a net baryon rapidity density of $d N_{B} / d y \approx 20-35$ and specific entropy of $s / \rho_{B} \approx 150-250$ in central $A u+A u$ at $\sqrt{s}=200 A \mathrm{GeV}$ at midrapidity. Perturbative QCD calculations of minijet production in leading logarithm approximation with a transverse momentum cutoff of $p_{0}=1 \mathrm{GeV}$ yield $s / \rho_{B}=150$, if a soft component with $s / \rho_{B}=50$ is assumed [16].

We will employ $d N_{B} / d y=25$ and $s / \rho_{B}=200$. For our choice of $\tau_{i}$ and equation of state, the resulting energy and baryon densities at midrapidity are $\epsilon_{i}=17 \mathrm{GeV} / \mathrm{fm}^{3}$ and $\rho_{i}=2.3 \rho_{0}$. The initial temperature and quark-chemical potentials follow as $T_{i}=300 \mathrm{MeV}$, $\mu_{q}=47 \mathrm{MeV}, \mu_{s}=0$, respectively. The higher specific entropy and lower net baryon number in $A u+A u$ collisions at RHIC energy as compared to SPS energy enhance the pion multiplicity at midrapidity by $\approx 50 \%$ [33]. Also, antibaryon to baryon ratios at midrapidity increase from $\approx 0.1$ to $\geq 0.5[33]$.

The total transverse energy at midrapidity on the hadronization hypersurface is 
$d E_{T} / d y=641 \mathrm{GeV}$, and decreases to $627 \mathrm{GeV}$ on the $T=130 \mathrm{MeV}$ isotherm. Thus, the isentropic expansion reduces the energy at midrapidity by almost a factor of two $\left(d E_{T} / d y\left(\tau_{i}\right)=1153 \mathrm{GeV}\right)$.

In an individual event, the transverse flow velocity field can be rather complicated [34], however, on average over many events it vanishes at $\tau=\tau_{i}$. The radial energy and net baryon densities are taken to be proportional to $\Theta\left(R_{T}-r_{T}\right) . R_{T}=6 \mathrm{fm}$ denotes the nuclear radius. To show the effect of initial pressure gradients on collective particle production at high $p_{T}$ we will also employ the "wounded nucleon" distribution

$$
\begin{aligned}
\epsilon\left(\tau_{i}, r_{T}\right) & =\epsilon_{i} f\left(r_{T}\right), \\
\rho_{B}\left(\tau_{i}, r_{T}\right) & =\rho_{i} f\left(r_{T}\right),
\end{aligned}
$$

with

$$
f\left(r_{T}\right)=\frac{3}{2} \sqrt{1-\frac{r_{T}^{2}}{R_{T}^{2}}} .
$$

$\epsilon_{i}, \rho_{i}$ denote the energy and net baryon densities as specified above for SPS and RHIC energies, respectively.

\section{Calculation of Particle Momentum Spectra}

It is generally unreasonable to assume that the fluid-dynamical description is valid in the whole forward lightcone. In some space-time regions the reaction rates between the particles are too low to maintain local thermal and/or chemical equilibrium. In hydrodynamical calculations one usually assumes that the transition between the fluid and the free streaming regime occurs within a space-time volume which is commonly approximated by a three-dimensional surface in four dimensional space-time [18, 35]. One then has to determine 
the parameters of the free-streaming gas, characterizing its momentum-space distribution, from the properties of the fluid (i.e. velocity, temperature, etc.) on the assumed freeze-out hypersurface. From the theoretical point of view, this complicated problem is not yet satisfactorily solved [36]. Problems may arise, in particular, if the momentum-space distribution functions on the two sides of the freeze-out hypersurface (fluid and free-streaming gas) differ. Also, on time-like parts of the freeze-out hypersurface (i.e. with space-like normal) negative contributions to the spectrum of frozen-out particles may arise. For high flow velocities through the hypersurface, however, these contributions are negligible.

Several attempts to understand and model the freeze-out can be found in the literature [35 37]. We will employ the description of Cooper and Frye [38, which has been used to calculate rapidity and transverse momentum spectra at AGS and SPS energies by several authors [8 12, 37, 39, 40]. For a cylindrically symmetric transverse expansion exhibiting longitudinal scaling flow the particle momentum spectra are given by

$$
\frac{d N}{d^{2} p_{T} d y}=\int_{0}^{1} d \zeta \int_{0}^{2 \pi} d \phi \int_{-\infty}^{\infty} d \eta r_{T} \tau\left(p_{T} \cos \phi \frac{d \tau}{d \zeta}-m_{T} \cosh (y-\eta) \frac{d r_{T}}{d \zeta}\right) f\left(p_{\mu} u^{\mu}\right)
$$

where $p^{\mu}=\left(m_{T} \cosh y, p_{T} \sin \chi, p_{T} \cos \chi, m_{T} \sinh y\right)$, and $u^{\mu}=\gamma_{T}\left(\cosh \eta, v_{T} \sin (\phi-\right.$ $\left.\chi), v_{T} \cos (\phi-\chi), \sinh \eta\right)$ denote the particle four-momentum and the fluid four-velocity, respectively. Thus, the direction of the particle momentum in the transverse plane is determined by the angle $\chi$, while the relative angle between $\vec{p}_{T}$ and the transverse flow velocity, $\vec{v}_{T}$, is denoted by $\phi . \zeta \in[0,1]$ parametrizes the hypersurface (counter-clockwise) on which the momentum distribution of particles is to be calculated, such that $r_{T}(\zeta)$ and $\tau(\zeta)$ specify the space-time points on the hypersurface. Since we focus on particles with $m_{T} \gg T_{f o}$, we approximate the phase-space distribution function $f$ by a Boltzmann distribution, and also

$$
\exp \left(-\frac{\gamma_{T} m_{T}}{T} \cosh (y-\eta)\right) \approx \sqrt{\frac{2 \pi T}{\gamma_{T} m_{T}}} \delta(y-\eta) \exp \left(-\frac{\gamma_{T} m_{T}}{T}\right)
$$


The thermal smearing of the rapidity distribution is thus approximated by the square-root factor. The quality of this approximation is less good for low- $p_{T}$ pions which are therefore not considered here.

For SPS energies, we calculate the transverse momentum spectra on the $T=130 \mathrm{MeV}$ isotherm. This turns out to reproduce the measured spectra of $\pi, p, \phi, \Lambda$, and $d$ reasonably well, cf. below. Also, this choice is in line with freeze-out temperatures extracted by others [2, 1 , 5, 9, 11].

Even without a detailed calculation of the reaction rates between various hadron species as a function of temperature and chemical potentials (cf. [41] for such a study and [37] for a first step to include it into the fluid-dynamical model) one might speculate that the freezeout temperature might be higher at RHIC than at SPS. The reason is that the lower net baryon density has to be compensated for by an increase in the meson density, in order that the reaction rates be large enough to maintain local thermodynamical equilibrium. We shall thus calculate the particle momentum distributions at RHIC on the hypersurface where the fluid enters the hadronic phase円, $\lambda=0 .\left(\lambda \equiv V^{Q G P} / V^{\text {tot }}\right.$ denotes the fraction of quark-gluon plasma within the mixed phase.) The results are not changed if a $T=160 \mathrm{MeV}$ isotherm is employed instead. To explore the range of uncertainty, however, we shall also show results for freeze-out at $T=130 \mathrm{MeV}$.

\footnotetext{
${ }^{1}$ Note that, for the EoS used here, hadronization proceeds via a shock wave [21,42]. In practice, we evaluate eq. (9) on the hypersurface corresponding to a small negative $\lambda=-0.05$. This ensures that the fluid properties (velocity, temperature etc.) are those of hadronic matter, located either on or behind the shock front.
} 


\section{RESULTS}

\section{A. Space-Time Evolution}

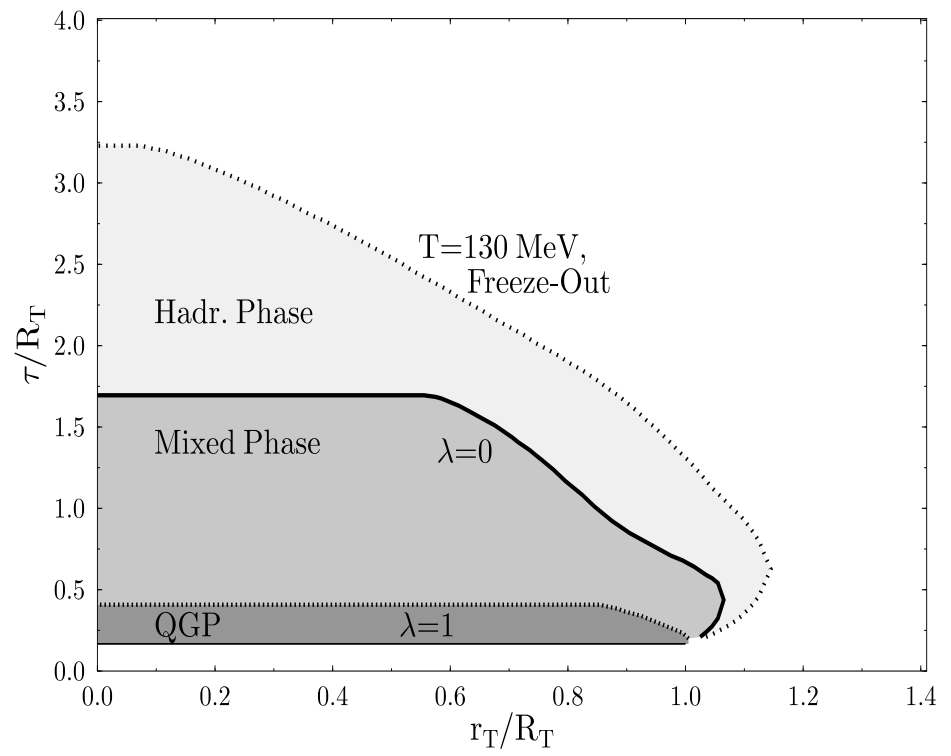

FIG. 1. Hypersurfaces corresponding to $\lambda=1$ (boundary between pure QGP and mixed phase), $\lambda=0$ (boundary between mixed phase and pure hadron phase), and the freeze-out isotherm $T=130 \mathrm{MeV}$; for central $\mathrm{Pb}+\mathrm{Pb}$ collisions at SPS.

Figures 11 and 2 show the hypersurfaces where the pure QGP and the mixed phase end, respectively, as well as the $T=130 \mathrm{MeV}$ isotherm. One observes a qualitative change in the space-time evolution. When comparing the evolution at SPS and RHIC, one finds that due to the high initial entropy density the QGP phase lives almost twice as long at RHIC than at SPS. Therefore, the transverse rarefaction wave can propagate further into the quark-gluon fluid and accelerate it. This leads to larger transverse flow of matter entering the mixed phase at RHIC than at SPS. Due to this effect the hadronization surface $(\lambda=0)$ extends to larger $r_{T}$. Note also that at SPS the fluid spends $\approx 50 \%$ of its lifetime in the hadronic 
phase, while at RHIC QGP and mixed phase occupy a much larger space-time volume. For comparison, we have also shown the $T=130 \mathrm{MeV}$ isotherm for $A u+A u$ reactions at RHIC.

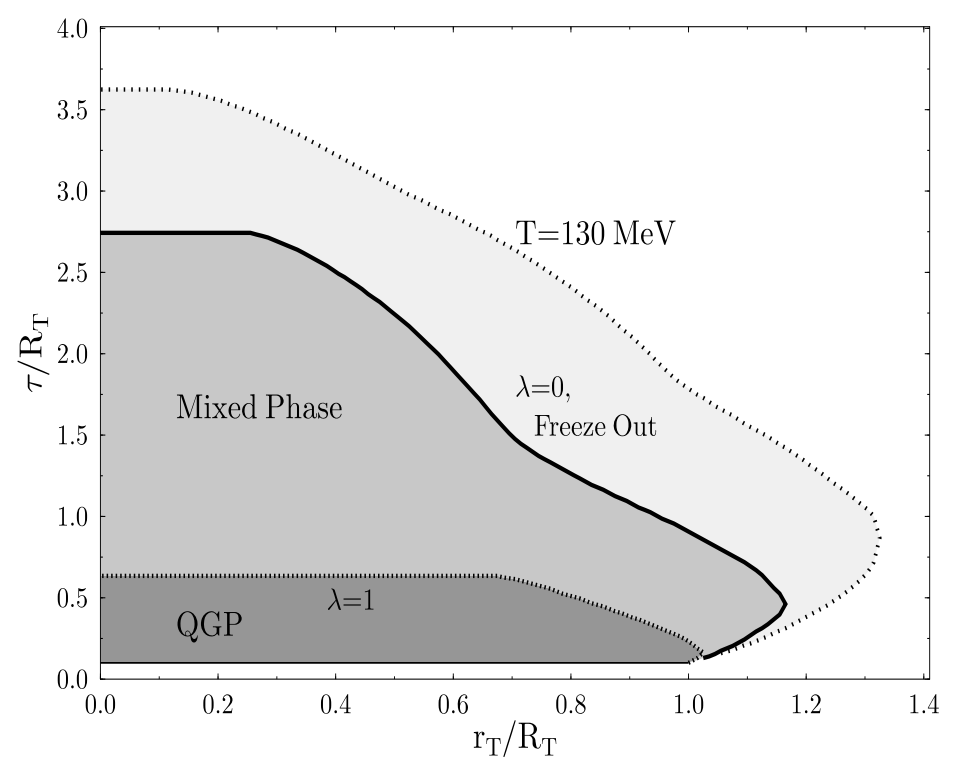

FIG. 2. Hypersurfaces corresponding to $\lambda=1$ (boundary between QGP and mixed phase), and the freeze-out hypersurface $\lambda=0$ (boundary between mixed phase and pure hadron phase); for central $A u+A u$ collisions at RHIC.

For our EoS, which contains all well-established hadrons up to mass $2 \mathrm{GeV}$, hadronization is completed at $\tau=1.7 R_{T}=10 \mathrm{fm}$ (SPS) resp. $\tau=2.7 R_{T}=16 \mathrm{fm}$ (RHIC). The values of the lifetime of the mixed phase obtained here agree with those predicted in ref. [20] for the case where the ratio of degrees of freedom in the QGP and hadronic phase is 3, cf. Fig. 12c in [20]. If only $\pi$ or $\pi, \eta, \rho, \omega$ mesons are considered in the hadron gas, complete hadronization takes considerably longer at RHIC, $\tau=6 R_{T}=36 \mathrm{fm}$ [20] and $\tau=30 \mathrm{fm}$ [43], respectively (cf. also refs. [9-11,40]). The back-reaction of the freeze-out on the dynamics of the fluid could further speed up hadronization by up to $30 \%$ [33]. 


\section{B. Transverse Momentum Spectra of Hadrons and Clusters}

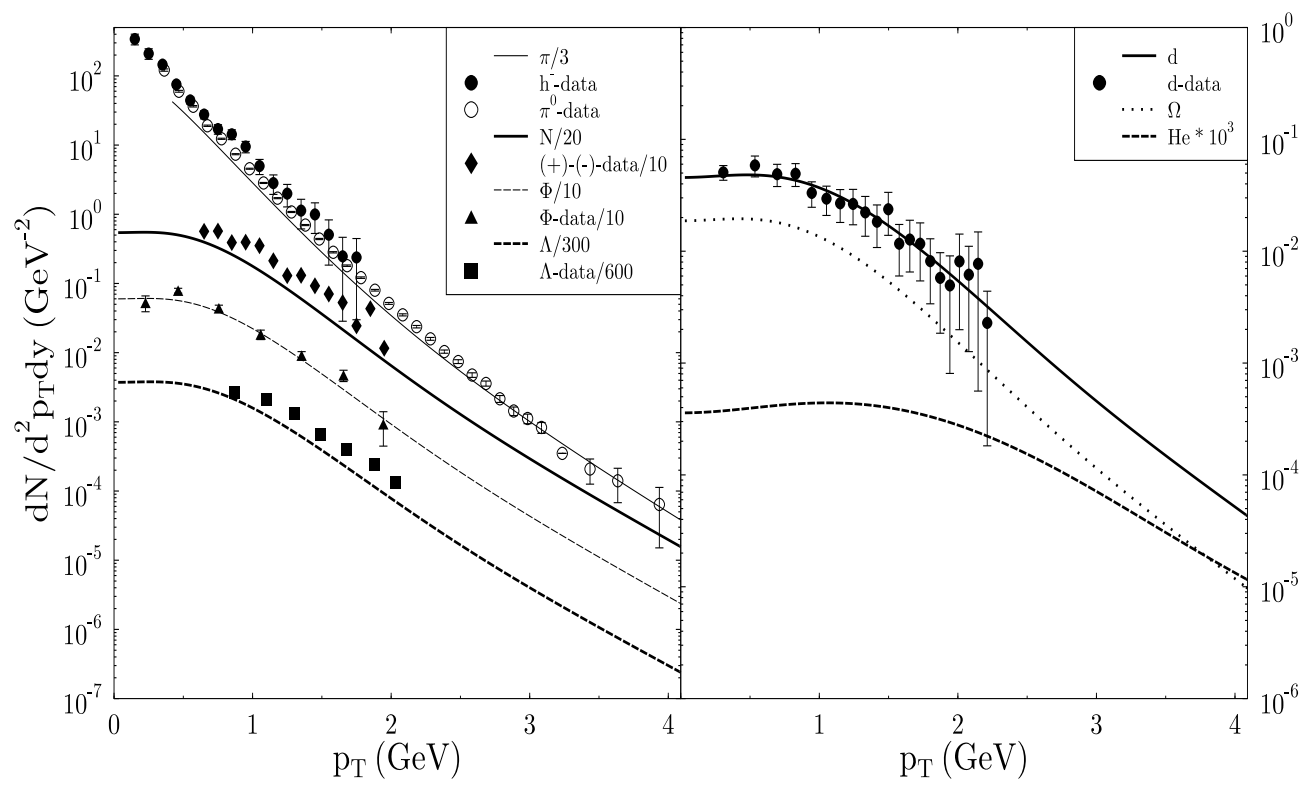

FIG. 3. Transverse momentum spectra of direct thermal hadrons at midrapidity (central $\mathrm{Pb}+\mathrm{Pb}$ collisions at SPS), calculated on the $T=130 \mathrm{MeV}$ isotherm. A step function has been employed for the initial transverse energy and baryon density profiles.

Figure 3 compares our calculated $p_{T}$ spectra with $h^{-},(+)-(-)$[1], $\phi, d$ [2], and $\Lambda$ [4] data of NA49. The additional factor $1 / 2$ in the $\Lambda$ data as compared to our calculation approximately corrects for the $\Sigma^{0}$ s, which can experimentally not be separated from the $\Lambda$ s. The preliminary $\pi^{0}$ data obtained by the WA98 collaboration are also shown [45] (see also the data obtained by the CERES collaboration [46]). Both the calculated spectra as well as the experimental data are absolute numbers without any arbitrary normalization factor. The reason for the underpredicted multiplicities of pions, protons, and lambdas is the fact 
that we have not included post-freeze-out resonance decays?2. Also, we have not explored effects of an isospin asymmetric initial state.

The shapes of the spectra, however, are well reproduced. In particular, one can see the broadening of the $p_{T}$ distribution as the particle mass increases, and the "shoulderarm" structure that develops in the spectra of the heavy clusters. This behavior emerges naturally in the presence of collective transverse flow [7, 12,47,48, and has already been discovered experimentally at the BEVALAC 49] and at the AGS [3].

Note also that due to collective transverse flow the spectra at high $p_{T}$ are not exponentials. The inverse slope of the thermal pions and nucleons in the region $1 \mathrm{GeV}<p_{T}<2 \mathrm{GeV}$ is $T_{\pi}^{*}=195 \mathrm{MeV}, T_{N}^{*}=220 \mathrm{MeV}$, and increases to $T_{\pi}^{*}=255 \mathrm{MeV}, T_{N}^{*}=270 \mathrm{MeV}$, respectively, at higher transverse momentum, $2 \mathrm{GeV}<p_{T}<3 \mathrm{GeV}$. This effect is even stronger in $A u+A u$ at RHIC, see below.

The apparent temperature of high- $p_{T}$ pions agrees well with the preliminary result of the WA98 collaboration, $T_{\pi}^{*}=250 \mathrm{MeV}$ at $p_{T}=2 \mathrm{GeV}$ 45. This holds also for the multiplicity of $\pi^{0} \mathrm{~S}$ with $p_{T}>2 \mathrm{GeV}$ at midrapidity; while we obtain $0.32 / 3=0.11$ (excluding $\pi^{0} \mathrm{~S}$ from post-freeze-out resonance decays) the WA98 collaboration found $0.138 \pm 0.05$ (preliminary) $\pi^{0} \mathrm{~s}$ in this region of momentum space. In contrast to the arguments of ref. 550 it can thus not be excluded that the measured high- $p_{T}$ pions can be described within a fluid-dynamical model.

\footnotetext{
${ }^{2}$ The total multiplicities, including feeding from resonance decays and assuming chemical freezeout at the phase boundary were calculated e.g. in refs. $4,28,33$.
} 


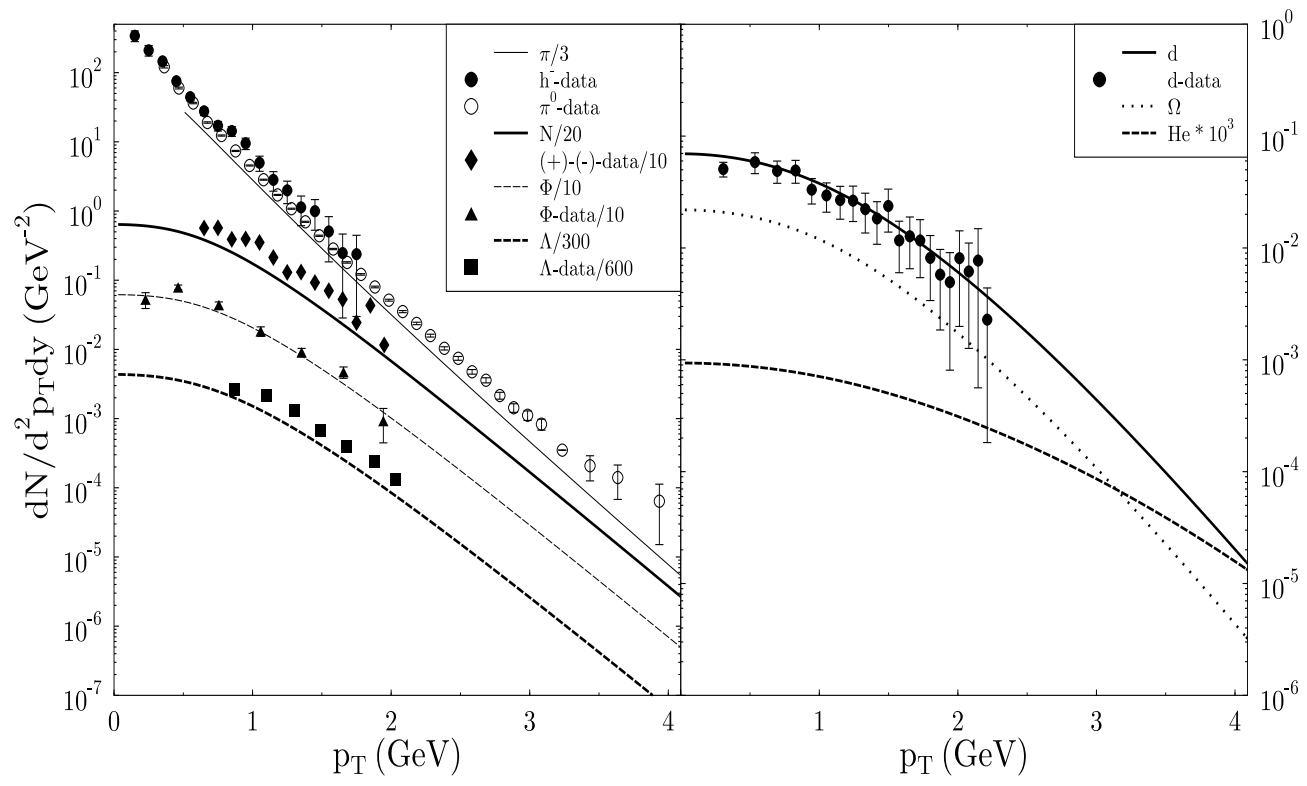

FIG. 4. Transverse momentum spectra of direct thermal hadrons at midrapidity (central $\mathrm{Pb}+\mathrm{Pb}$ collisions at SPS), calculated on the $T=130 \mathrm{MeV}$ isotherm. A "wounded nucleon" distribution has been employed for the initial transverse energy and baryon density.

We emphasize, however, that collective particle production at high $p_{T}$ shows some sensitivity to the initial conditions. Employing a "wounded nucleon" distribution, cf. eq. (17), the pion spectrum (on the $T=130 \mathrm{MeV}$ hypersurface) above $p_{T}=1 \mathrm{GeV}$ is again closer to an exponential, cf. Fig. \$. On the other hand, boost non-invariant initial conditions [9 11], or initial transverse velocity gradients [34], can again enhance collective transverse expansion. In view of these uncertainties, we argue that collective hadron production above $p_{T}=1 \mathrm{GeV}$ cannot be definitely excluded. 


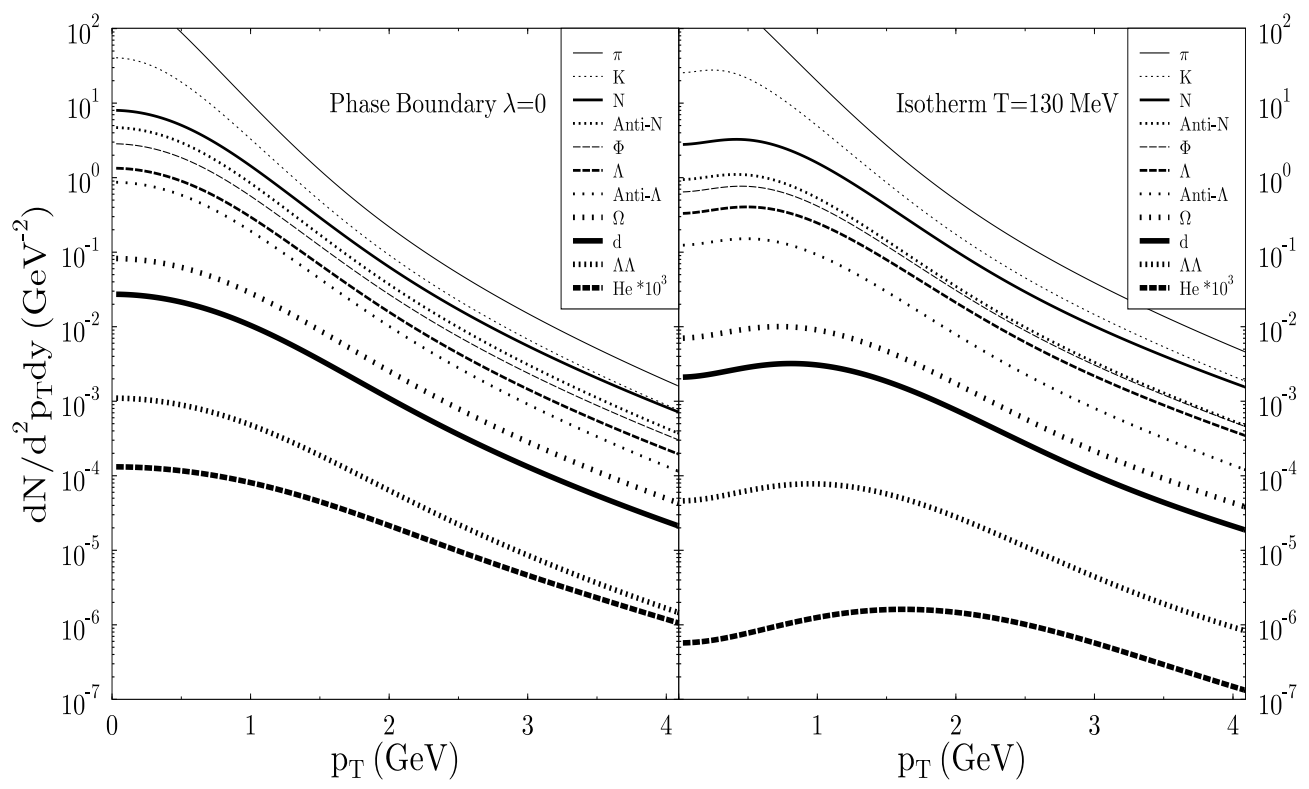

FIG. 5. Transverse momentum spectra of direct thermal hadrons at midrapidity (central $A u+A u$ collisions at RHIC), as calculated on the $\lambda=0$ and $T=130 \mathrm{MeV}$ hypersurfaces, respectively. A step function has been employed for the initial transverse energy and baryon density profiles.

Figure 5 shows the $p_{T}$ spectra for RHIC energy as calculated on the $\lambda=0$ and $T=$ $130 \mathrm{MeV}$ hypersurfaces. The $\Lambda \Lambda$ is a hypothetical bound state of two $\Lambda$ baryons for which we assume a binding energy of $30 \mathrm{MeV}$. If freeze-out occurred later, e.g. on the $T=130 \mathrm{MeV}$ isotherm like in $\mathrm{Pb}+\mathrm{Pb}$ at SPS, the resulting spectra are of course "stiffer", since collective flow is significantly stronger than on the $\lambda=0$ hypersurface. This leads to much broader $p_{T}$ distributions for the heavy hadrons. Observe also that the number of pions at $p_{T}=4 \mathrm{GeV}$ increases by a factor of 3 .

We would like to emphasize also another qualitative difference in the dynamics at SPS and RHIC, where the space-time volume of the hadronic phase might be smaller. At RHIC, 
the outer part of the fluid $\left(r_{T}>0.7 R_{T}\right.$, cf. Fig. 2) is strongly accelerated by the rarefaction wave which moves into the QGP. However, once longitudinal expansion has cooled the core of the fluid into the phase coexistence region, the transverse rarefaction wave is stopped abruptly, since the velocity of sound in the mixed phase is small. Thus, if the space-time volume of the purely hadronic phase is small, the interior part of the fluid will remain more or less at rest. We consequently expect two different slopes in the $p_{T}$ distributions. This is confirmed by Fig. 5, left panel. In the range $1 \mathrm{GeV}<p_{T}<2 \mathrm{GeV}$, the apparent temperature of thermal pions and nucleons is $T_{\pi}^{*}=220 \mathrm{MeV}$ and $T_{N}^{*}=233 \mathrm{MeV}$, respectively; at higher $p_{T}, 2 \mathrm{GeV}<p_{T}<3 \mathrm{GeV}$, the inverse slopes increase by roughly $100 \mathrm{MeV}$, to $T_{\pi}^{*}=325 \mathrm{MeV}$, and $T_{N}^{*}=335 \mathrm{MeV}$ ! Note that the difference is much larger than in $\mathrm{Pb}+\mathrm{Pb}$ at SPS, although the average transverse flow velocity (on the $\lambda=0$ hypersurface) is smaller than on the $T=130 \mathrm{MeV}$ isotherm at SPS (see below).

\footnotetext{
${ }^{3}$ Parametrization of collective transverse flow in terms of a simple radial velocity profile 48 is therefore not possible on the $\lambda=0$ hypersurface. This is in contrast to freeze-out occurring later, e.g. on the $T=130 \mathrm{MeV}$ isotherm.
} 


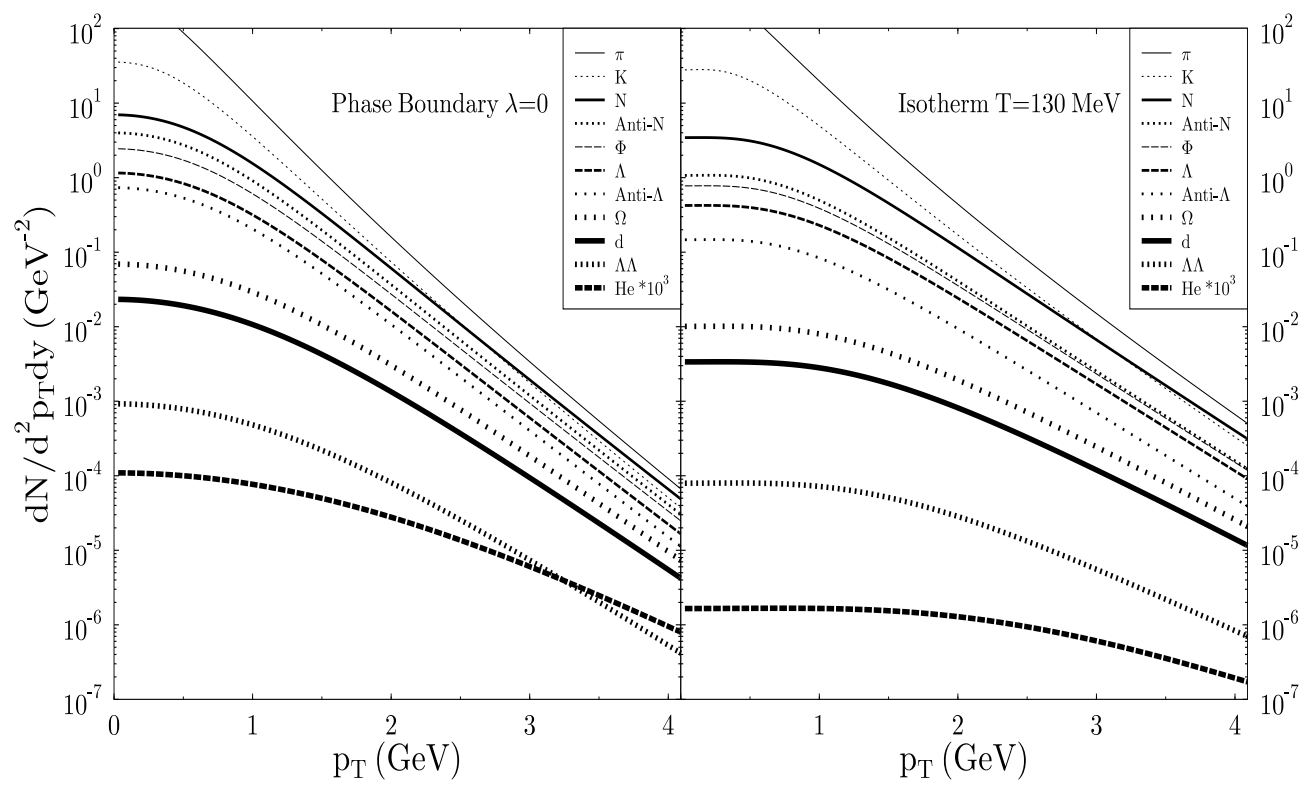

FIG. 6. Transverse momentum spectra of direct thermal hadrons at midrapidity (central $A u+A u$ collisions at RHIC), as calculated on the $\lambda=0$ and $T=130 \mathrm{MeV}$ hypersurfaces, respectively. A "wounded nucleon" distribution has been employed for the initial transverse energy and baryon density.

However, if the initial transverse energy and baryon density distributions exhibit smaller gradients, this kink in the spectra is washed out, cf. Fig. 6. For the "wounded nucleon" distribution, eq. (7), the very fast tails in the velocity distribution on the hypersurface are absent, thus reducing the number of hadrons at $p_{T}>2 \mathrm{GeV}$. Figures 5 and 6 show the possible range of collective particle production at high transverse momenta. On the other hand, we have found that the two initial density distributions lead to average transverse momenta that differ by $\leq 10 \%$. Therefore, we present results for $\left\langle p_{T}\right\rangle$ only for the sharp density profile. 


\section{Mean Transverse Velocities and Momenta}

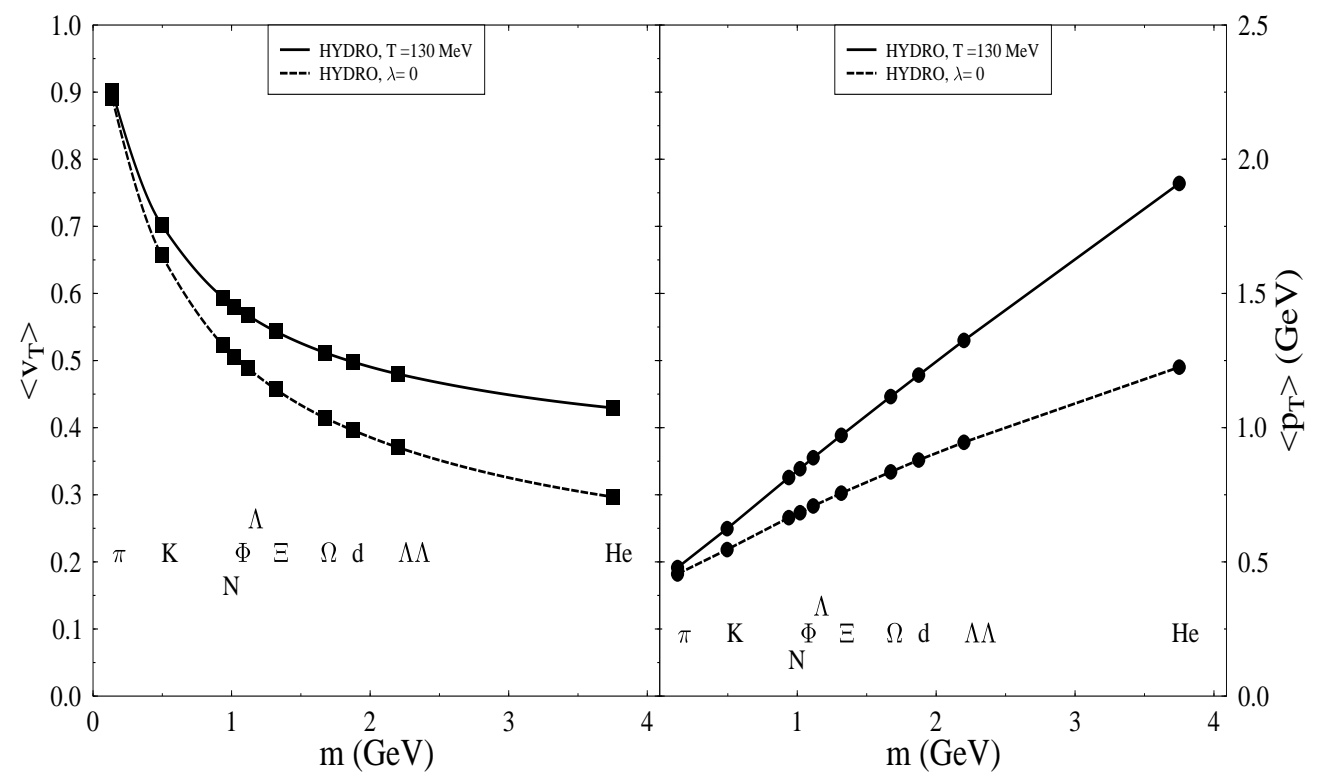

FIG. 7. Average transverse momenta and velocities (at midrapidity) of direct thermal hadrons on the boundary between mixed phase and pure hadron phase, $\lambda=0$, and on the $T=130 \mathrm{MeV}$ isotherm (for central $\mathrm{Pb}+\mathrm{Pb}$ collisions at SPS).

Figures 7 and 8 depict the average transverse momenta and velocities of various hadrons at midrapidity, as calculated from the $p_{T}$ distributions:

$$
\left(\left\langle p_{T}\right\rangle_{i},\left\langle v_{T}\right\rangle_{i}\right)=\frac{\int d^{2} p_{T}\left(p_{T}, p_{T} / p^{0}\right) \frac{d N_{i}}{d^{2} p_{T} d y}}{\int d^{2} p_{T} \frac{d N_{i}}{d^{2} p_{T} d y}} .
$$


One observes that due to the collective transverse expansion, $\left\langle p_{T}\right\rangle=m\left\langle\gamma_{T} v_{T}\right\rangle$ increases linearly with the particle mass predicted, NA49 measured $418 \pm 30 \mathrm{MeV}$ [1]. However, this is not astonishing since we have employed the Boltzmann approximation, and have also not included pions from postfreeze-out resonance decays. For all other (heavier) hadrons these effects on $\left\langle p_{T}\right\rangle$ are less significant.

On the other hand, $\left\langle v_{T}\right\rangle$ saturates with increasing $m$, since the thermal velocities $\sim \sqrt{T_{f o} / m}$ become negligible and $\langle v\rangle \approx v_{\text {flow }}$. Thus, clusters $(d, H e, \ldots)$ provide the opportunity to determine the transverse flow velocity directly [7,47], while the thermal averaging cannot be neglected for nucleons and especially for pions and kaons. To extract the collective flow from their $\left\langle p_{T}\right\rangle$ or $\left\langle v_{T}\right\rangle$ requires the knowledge of the freeze-out temperature, and moreover might be distorted by decays of resonances [5,48]. The average transverse flow velocity in $\mathrm{Pb}+\mathrm{Pb}$ at SPS is $\approx 0.3$ on the boundary between mixed and hadronic phase (i.e. $\lambda=0$ ), and increases to $\approx 0.4$ on the $T=130 \mathrm{MeV}$ isotherm. This is consistent with other fluid dynamical calculations [9 11, 37] and numbers extracted from fits of transverse momentum distributions [2, [4.5,48]. Our analysis does not support freeze-out temperatures of less than $100 \mathrm{MeV}$ at SPS [51], at least not at midrapidity.

\footnotetext{
${ }^{4}$ Note, however, that $\left\langle\gamma_{T} v_{T}\right\rangle$ does in principle depend on the particle mass, since it includes flow as well as thermal velocity components, cf. left panel of Fig. 17. Thus, the result $\left\langle p_{T}\right\rangle \propto m$ is not trivial.
} 


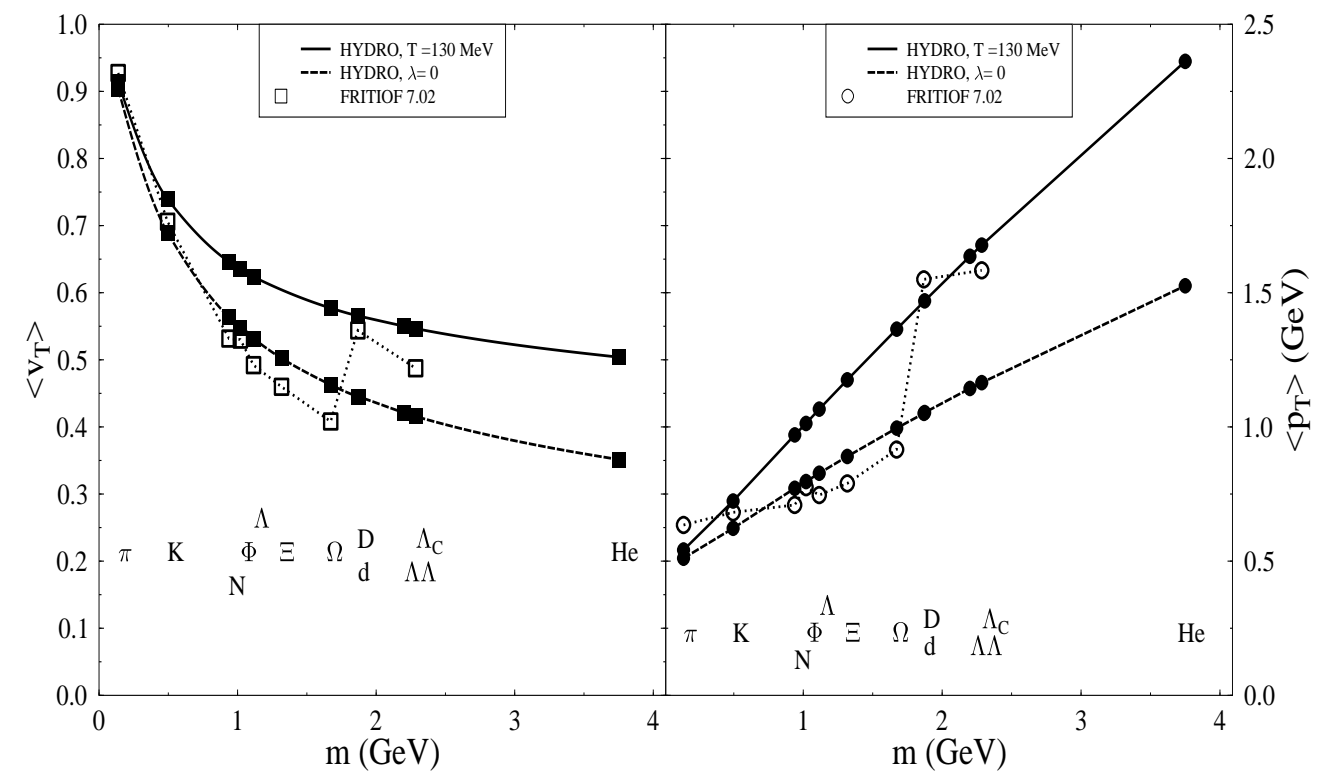

FIG. 8. Same as fig. 目 but for central $A u+A u$ collisions at RHIC. Open symbols depict the predictions of FRITIOF 7.02 .

Although the entropy per net baryon at RHIC is five times larger than at SPS, the transverse flow velocity increases only slightly. (In particular, the $\left\langle p_{T}\right\rangle$ of pions almost stagnates from SPS to RHIC.) This is due to the fact that the transverse flow cannot increase strongly in the expansion of the mixed phase, since the velocity of sound is very small [20, 22, 52]. Thus, hydrodynamics predicts that the transverse flow velocity at RHIC is comparable to that at SPS, if the EoS exhibits a first-order phase transition. If freeze-out at RHIC occurs as early as on the $\lambda=0$ hypersurface, the transverse flow velocity could even be smaller than at SPS, cf. Figs. 7 and 8 .

It is also interesting to note that both at SPS and RHIC a significant part of the transverse flow is already produced within the QGP and (to a smaller part also within) the mixed phase, 
compare the dashed curves in figs. 0 and 8 with the solid lines. This behavior is different to that observed within the RQMD 2.3 model, where the transverse flow of baryons emerges mainly from the late stage of the reaction [13]. The reason is that within RQMD 2.3 the hadron gas is not in kinetic equilibrium at times $t<5 \mathrm{fm}$, while we assume early kinetic equilibrium of the QGP in the central region, $\tau_{i}=1 \mathrm{fm}$.

The flow of the $\phi$ meson is closely related to this point. Even if $\phi$ mesons do not rescatter with surrounding hadronic or mixed phase matter, their $\left\langle p_{T}\right\rangle,\left\langle v_{T}\right\rangle$ or inverse slope in AA collisions should be affected by collective flow if a QGP with $s$ quarks in local thermal equilibrium is created. This is due to the fact that the transverse momenta of $s$ quarks are subject to a Doppler shift according to the collective flow velocity built up in the QGP phase. According to Fig. 7], $\left\langle p_{T}\right\rangle_{\phi} \approx 680 \mathrm{MeV}$ and $\left\langle v_{T}\right\rangle_{\phi} \approx 0.5$ at SPS, even if the $\phi$ 's decouple from collective flow immediately at the boundary between mixed and hadronic phase. Indeed, preliminary NA49 data [2] show that the $\phi$ roughly fits into the $p_{T}(m)$ systematics predicted by hydrodynamics, as already indicated by Fig. 3. Note that the "conventional" mechanism to generate $p_{T}$ in cascade models, i.e. resonance decay or color flux-tube fragmentation followed by additional rescattering with the measured free (or additive quark model) cross section, apparently works for $\pi, K, N, \Lambda$ but not for the $\phi$ 53 (cf. also the discussion in ref. [54]).

For comparison, we have also calculated the average transverse momentum and velocity of direct hadronsf (i.e. resonance decays were also switched off) at RHIC within FRITIOF 7.02 [17], cf. fig. 8. In this string model, secondary hadrons do not rescatter. Thus, collective transverse flow does not develop. One observes that even though hard scatterings

\footnotetext{
${ }^{5}$ Clusters $(d, \Lambda \Lambda$, and $H e)$ are not computed within this model.
} 
are taken into account, the $\left\langle p_{T}\right\rangle$ of $N, \phi, \Lambda$, and $\Omega$ is not higher than in case of hydrodynamic expansion with freeze-out on the $\lambda=0$ hypersurface. This difference would be even bigger if collective flow set in earlier (remember that we assume $\tau_{i}=0.6 \mathrm{fm}$ ).

Only the $\left\langle p_{T}\right\rangle$ of charmed hadrons, $D$ meson and $\Lambda_{C}(2285)$ baryon $]$, is strongly enhanced by (semi-)hard scatterings, and is close to the solid line in fig. 8. These very heavy hadrons are produced by fragmentation of semi-hard jets and acquire high transverse momentum. Thus, in FRITIOF 7.02 the $\left\langle p_{T}\right\rangle$ of various hadrons does not increase linearly with mass but depends strongly on the sensitivity to (semi-)hard scatterings, and thus to the flavor content. In contrast, if $D$ mesons thermalize [55] and follow the collective transverse flow, their $\left\langle p_{T}\right\rangle$ is "pulled" back onto the linear $\left\langle p_{T}\right\rangle(m)$ systematics.

\section{SUMMARY}

In this paper we have investigated the collective evolution of the central rapidity region with initial conditions as expected for heavy-ion collisons at CERN-SPS and BNL-RHIC energies. Even in the case where the phase transition (assumed to be of first order) from the plasma of $u, d, s$ quarks and gluons to the hadron gas proceeds in local thermodynamical equilibrium, collective transverse expansion and equilibration of heavy mesons and (anti)baryons reduces the hadronization time to $\leq 3 R_{T}$ (for $A u$ nuclei). Nevertheless, at RHIC, the lifetimes of the QGP and the mixed phase are significantly longer than at SPS. However, at SPS the midrapidity region evolves further into the hadronic phase, which occupies a

\footnotetext{
${ }^{6}$ Pions and kaons are not very sensitive to the dynamical evolution and are left out of the discussion for the moment.
} 
larger space-time volume than the QGP or mixed phase. As a consequence, the transverse flow velocity and the mean transverse momentum of the particles increase substantially during this stage of the reaction. Without this "hadronic afterburner" the hydrodynamical model as discussed in this paper would not describe the measured single-particle transverse momentum distributions.

At RHIC, one might expect a higher freeze-out temperature than at SPS, since the baryon density is smaller. If, for example, freeze-out occurs directly after hadronization is complete (i.e. on the $\lambda=0$ hypersurface), the average transverse flow velocity turns out to be similar or even smaller than at SPS.

We have calculated the transverse momentum distributions of various hadrons up to $p_{T}=4 \mathrm{GeV}$ on the $T=130 \mathrm{MeV}$ isotherm and on the $\lambda=0$ hypersurface for SPS and RHIC, respectively. Large gradients in the initial transverse density distributions (e.g. a sharp step function) can strongly enhance particle production at high $p_{T}$. The recently observed $\pi^{0}$ distribution (up to $p_{T}=4 \mathrm{GeV}$ ) in $\mathrm{Pb}+\mathrm{Pb}$ at $\sqrt{s}=18 \mathrm{~A} \mathrm{GeV}$ can in principle be described by a hydrodynamic expansion. However, the high- $p_{T}$ tails of the hadron distributions (above $p_{T}=2 \mathrm{GeV}$ ) are sensitive to the initial pressure gradients.

Within hydrodynamics the mean transverse momentum of hadrons increases linearly with the particle mass and is independent of other quantum numbers (provided the corresponding hadron species is in local thermal equilibrium). Heavy hadrons and clusters, if identified, allow for a direct measurement of the collective transverse expansion velocity, without requesting knowledge of freeze-out temperatures.

Finally, we have compared the fluid-dynamical results to those obtained within the string model FRITIOF 7.02. Due to the increasing "sensibility" on (semi-)hard processes, the $\left\langle p_{T}\right\rangle$ of hadrons with $c$ quarks increases more strongly than the $\left\langle p_{T}\right\rangle$ of hadrons with only light (anti- 
quarks. Consequently, there is a strongly nonlinear relation between the mean transverse momentum and the particle mass within this model.

\section{ACKNOWLEDGMENTS}

We thank L. Gerland, M. Gyulassy, G. Kunde, T. Peitzmann, K. Redlich, and C. Spieles for many helpful discussions, C. Bormann, J. Günther, G. Roland, and C. Struck for providing the NA49 data, and T. Peitzmann for communicating the preliminary WA98 data. A.D. gratefully acknowledges a postdoctoral fellowship by the German Academic Exchange Service (DAAD).

[1] T. Wienold and the NA49 collaboration, Nucl. Phys. A610, 76c (1996); P.G. Jones and the NA49 collaboration, ibid p. 188c; we have used more recent data from J. Günther, PhD thesis, Univ. Frankfurt 1998

[2] G. Roland et al., (NA49 collaboration), Proceedings of "Quark Matter 1997", to be published in Nucl. Phys. A

[3] L. Ahle et al., (E802 collaboration), Nucl. Phys. A610, 139c (1996); J. Barette et al., (E877 collaboration), ibid p. $153 \mathrm{c}$

[4] P. Braun-Munzinger, J. Stachel, J.P. Wessels, and N. Xu, Phys. Lett. B 365, 1 (1996)

[5] H. Ströbele, Nucl. Phys. A610, 102c (1996); N. Xu et al., ibid p. 175c; J. Stachel, ibid p. 509c; S. Esumi, S. Chapman, H. van Hecke, and N. Xu, Phys. Rev. C 55, R2163 (1997); I. G. Bearden et al., (NA44 collaboration), Phys. Rev. Lett. 78, 2080 (1997) 
[6] L. Bravina, N.S. Amelin, L.P. Csernai, P. Levai, and D. Strottman, Nucl. Phys. A566, 461c (1994); L. Bravina, L.P. Csernai, P. Levai, and D. Strottman, Phys. Rev. C 50, 2161 (1994)

[7] R. Mattiello, A. Jahns, H. Sorge, H. Stöcker, and W. Greiner, Phys. Rev. Lett. 74, 2180 (1995); R. Mattiello, H. Sorge, H. Stöcker, and W. Greiner, Phys. Rev. C 55, 1443 (1997)

[8] N. Arbex, U. Ornik, M. Plümer, and R.M. Weiner, Phys. Rev. C 55, 860 (1997)

[9] J. Sollfrank, P. Huovinen, M. Kataja, P.V. Ruuskanen, M. Prakash, and R. Venugopalan, Phys. Rev. C 55, 392 (1997)

[10] J. Sollfrank, P. Huovinen, and P.V. Ruuskanen, nucl-th/9801023

[11] B.R. Schlei, U. Ornik, M. Plümer, D. Strottman, and R.M. Weiner, Phys. Lett. B 376, 212 (1996); B.R. Schlei, Heavy Ion Phys. 5, 403 (1997)

[12] J. Alam, J. Cleymans, K. Redlich, and H. Satz, nucl-th/9707042

[13] H. van Hecke, H. Sorge, and N. Xu, hep-ph/9804035

[14] B. Müller and X.N. Wang, Phys. Rev. Lett. 68, 2437 (1992); E. Shuryak, ibid p. 3270; K. Geiger, Phys. Rev. D 46, 4965 (1992); E. Shuryak and L. Xiong, Phys. Rev. Lett. 70, 2241 (1993); T.S. Biro, E. van Doorn, B. Müller, M.H. Thoma, and X.N. Wang, Phys. Rev. C 48, 1275 (1993); B. Kämpfer and O.P. Pavlenko, Z. Phys. C 62, 491 (1994); K.J. Eskola and X.N. Wang, Phys. Rev. D 49, 1284 (1994)

[15] K. Geiger and J.I. Kapusta, Phys. Rev. D 47, 4905 (1993)

[16] K.J. Eskola and K. Kajantie, Z. Phys. C 75, 515 (1997)

[17] H. Pi, Comput. Phys. Comm. 71, 173 (1992); B. Andersson, G. Gustafson, and H. Pi, Z. Phys. 
C 57, 485 (1993); G. Gustafson, Nucl. Phys. A566, 233c (1994)

[18] L.D. Landau and E.M. Lifshitz, "Fluid Mechanics", Pergamon Press, New York, 1959

[19] K. Kajantie and L. McLerran, Phys. Lett. B 119, 203 (1982); Nucl. Phys. B214, 261 (1983); J.D. Bjorken, Phys. Rev. D 27, 140 (1983); G. Baym, B.L. Friman, J.P. Blaizot, M. Soyeur, and W. Czyz, Nucl. Phys. A407, 541 (1983); K. Kajantie, R. Raitio, and P.V. Ruuskanen, Nucl. Phys. B222, 152 (1983)

[20] D.H. Rischke and M. Gyulassy, Nucl. Phys. A608, 479 (1996)

[21] D.H. Rischke, S. Bernard, and J.A. Maruhn, Nucl. Phys. A595, 346 (1995)

[22] D.H. Rischke and M. Gyulassy, Nucl. Phys. A597, 701 (1996)

[23] E. Laermann, Nucl. Phys. A610, 1c (1996)

[24] A. Chodos, R.L. Jaffe, K. Johnson, C.B. Thorn, and V.F. Weisskopf, Phys. Rev. D 9, 3471 (1974)

[25] L.A. Winckelmann et al., Nucl. Phys. A610, 116c (1996)

[26] J. Kapusta, L. McLerran, and D.K. Srivastava, Phys. Lett. B 283, 145 (1992)

[27] M. Reiter, A. Dumitru, J. Brachmann, J.A. Maruhn, H. Stöcker, and W. Greiner, nuclth/9806010

[28] C. Spieles, H. Stöcker, and C. Greiner, Eur. Phys. J. C2, 351 (1998)

[29] T. Alber et al., (NA49 collaboration), Phys. Rev. Lett. 75, 3814 (1995)

[30] T. Schönfeld, H. Stöcker, W. Greiner, and H. Sorge, Mod. Phys. Lett. A 8, 2631 (1993) 
[31] H. Stöcker et al., in Proc. 4th Int. Workshop "Relativ. Aspects of Nucl. Phys.", Rio de Janeiro 1995 (Brazil) (eds. T. Kodama, K.C. Chung, Y. Hama, G. Odyniec, H. Ströbele, C.-Y. Wong), World Scientific, Singapore, 1996, p. 437

[32] S.E. Vance, M. Gyulassy, and X.N. Wang, nucl-th/9802036

[33] A. Dumitru, C. Spieles, H. Stöcker, and C. Greiner, Phys. Rev. C 56, 2202 (1997)

[34] M. Gyulassy, D.H. Rischke, and B. Zhang, Nucl. Phys. A613, 397 (1997)

[35] J.A. Maruhn and W. Greiner, in "Treatise on Heavy-Ion Science", Vol. 4, ed. D.A. Bromley, Plenum Press, New York/London 1985, p. 565; R.B. Clare and D. Strottman, Phys. Rep. 141, $177(1986)$

[36] Y. Sinyukov, Z. Phys. C 43, 401 (1989); L. Bravina, I.N. Mishustin, N.S. Amelin, J.P. Bondorf, and L.P. Csernai, Phys. Lett. B 354, 196 (1995); F. Grassi, Y. Hama, and T. Kodama, Phys. Lett. B 355, 9 (1995); Z. Phys. C 73, 153 (1996); S. Bernard, J.A. Maruhn, W. Greiner, and D.H. Rischke, Nucl. Phys. A605, 566 (1996); K.A. Bugaev, Nucl. Phys. A606, 559 (1996); L.P. Csernai, Z.I. Lazar, and D. Molnar, Heavy Ion Phys. 5, 467 (1997)

[37] C.M. Hung and E. Shuryak, Phys. Rev. C 57, 1891 (1998)

[38] F. Cooper and G. Frye, Phys. Rev. D 10, 186 (1974); F. Cooper, G. Frye, and E. Schonberg, Phys. Rev. D 11, 192 (1975)

[39] D. Kusnezov and G. Bertsch, Phys. Rev. C 40, 2075 (1989); J. Bolz, U. Ornik, and R.M. Weiner, Phys. Rev. C 46, 2047 (1992); A. Dumitru, U. Katscher, J.A. Maruhn, H. Stöcker, W. Greiner, and D.H. Rischke, Phys. Rev. C 51, 2166 (1995)

[40] J. Cleymans, K. Redlich, and D.K. Srivastava, Phys. Rev. C 55, 1431 (1997) 
[41] M. Prakash, M. Prakash, R. Venugopalan, and G. Welke, Phys. Rep. 227, 321 (1993)

[42] J.P. Blaizot and J.Y. Ollitrault, Phys. Rev. D 36, 916 (1987); K.A. Bugaev, M.I. Gorenstein, B. Kämpfer, and V.I. Zhdanov, Phys. Rev. D 40, 2903 (1989)

[43] J. Alam, D.K. Srivastava, B. Sinha, and D.N. Basu, Phys. Rev. D 48, 1117 (1993)

[44] C. Bormann et al., (NA49 collaboration), NA49 Note number 124, to be published in the proceedings of "Strangeness in Quark Matter 97", Santorini, Greece, April 14-18, 1997

[45] T. Peitzmann et al., (WA98 collaboration), Proceedings of "Quark Matter 1997", to be published in Nucl. Phys. A, and private communication

[46] F. Ceretto et al., (CERES collaboration), Proceedings of "Quark Matter 1997", to be published in Nucl. Phys. A

[47] P.J. Siemens and J.O. Rasmussen, Phys. Rev. Lett. 42, 880 (1979); H. Stöcker, A.A. Ogloblin, and W. Greiner, Z. Phys. A 303, 259 (1981)

[48] K. Lee, U. Heinz, and E. Schnedermann, Z. Phys. C 48, 525 (1990); E. Schnedermann, J. Sollfrank, and U. Heinz, Phys. Rev. C 48, 2462 (1993); E. Schnedermann, and U. Heinz, Phys. Rev. C 50, 1675 (1994)

[49] S. Nagamiya et al., Phys. Rev. C 24, 971 (1981); K.H. Kampert, J. Phys. G 15, 691 (1989)

[50] X.-N. Wang, hep-ph/9804384

[51] J.R. Nix, D. Strottman, H.W. van Hecke, B.R. Schlei, J.P. Sullivan, and M.J. Murray, nuclth/9801045

[52] M. Kataja, P.V. Ruuskanen, L.D. McLerran, and H. von Gersdorff, Phys. Rev. D 34, 2755 
(1986); C.M. Hung and E. Shuryak, Phys. Rev. Lett. 75, 4003 (1995)

[53] M. Bleicher, C. Spieles, C. Ernst, L. Gerland, S. Soff, H. Stöcker, W. Greiner, and S.A. Bass, hep-th/9803346

[54] H. Sorge, Nucl. Phys. A630, 522 (1998)

[55] B. Svetitsky and A. Uziel, Phys. Rev. D 55, 2616 (1997) 